\title{
Impact of adequate empirical combination therapy on mortality from bacteremic Pseudomonas aeruginosa pneumonia
}

\author{
So-Youn Park', Hyun Jung Park', Song Mi Moon ${ }^{1}$, Ki-Ho Park', Yong Pil Chong ${ }^{1}$, Mi-Na Kim², Sung-Han Kim',
} Sang-Oh Lee', Yang Soo Kim', Jun Hee Woo ${ }^{1}$ and Sang-Ho Choi ${ }^{1 *}$

\begin{abstract}
Background: Pseudomonas aeruginosa has gained an increasing amount of attention in the treatment of patients with pneumonia. However, the benefit of empirical combination therapy for pneumonia remains unclear. We evaluated the effects of adequate empirical combination therapy and multidrug-resistance in bacteremic Pseudomonas pneumonia on the mortality.

Methods: A retrospective cohort study was performed at the 2,700-bed tertiary care university hospital. We reviewed the medical records of patients with bacteremic pneumonia between January 1997 and February 2011. Patients who received either inappropriate or appropriate empirical therapy were compared by using marginal structural model. Furthermore, we investigated the direct impact of combination therapy on clinical outcomes in patients with monomicrobial bacteremic pneumonia.

Results: Among 100 consecutive patients with bacteremic Pseudomonas pneumonia, 65 patients were classified in the adequate empirical therapy group, 32 of whom received monotherapy and 33 combination therapy. In the marginal structural model, only inadequate therapy was significantly associated with 28-day mortality $(p=0.02)$, and multidrug-resistance was not a significant risk factor.

To examine further the direct impact of combination therapy, we performed a subgroup analysis of the 65 patients who received adequate therapy. Multivariate logistic regression analysis identified absence of septic shock at the time of bacteremia $(\mathrm{OR}, 0.07 ; 95 \% \mathrm{Cl}, 0.01-0.49 ; p=0.008)$, and adequate combination therapy $(\mathrm{OR}, 0.05 ; 95 \% \mathrm{Cl}$, 0.01-0.34; $p=0.002$ ) as variables independently associated with decreased all-cause 28-day mortality.
\end{abstract}

Conclusions: Our study suggests that adequate empirical combination therapy can decrease mortality in patients with bacteremic Pseudomonas pneumonia.

Keywords: Pseudomonas aeruginosa, Pneumonia, Combination treatment

\section{Background}

Pseudomonas aeruginosa has emerged as a significant nosocomial pathogen with a substantial frequency of multidrug resistance (MDR) [1]. Moreover pneumonia caused by $P$. aeruginosa is known to be associated with greater mortality than infection of other sites [2-5]. Hence, treatment of $P$. aeruginosa pneumonia poses a

\footnotetext{
* Correspondence: sangho@amc.seoul.kr

'Departments of Infectious Diseases, Asan Medical Center, University of Ulsan College of Medicine, 388-1 Pungnap-dong, Songpa-gu, Seoul, Republic of Korea

Full list of author information is available at the end of the article
}

great challenge to clinicians. Early studies, mainly of neutropenic patients with bacteremia, showed that mortality was decreased in patients receiving combination therapy [6]. However, the results of subsequent clinical studies on the effect of combination therapy on the treatment of severe $P$. aeruginosa infection have been conflicting [7-11]. These previous studies had significant limitations, including the enrollment of only small numbers of patients infected with different kinds of gramnegative bacilli and the lack of controls. Furthermore, the validity of diagnoses of $P$. aeruginosa pneumonia has itself been questioned because $P$. aeruginosa often 
colonizes the respiratory tract in hospital settings, and it is often difficult for clinicians to distinguish a colonizer from a true pathogen [2]. Thus, the isolation of P. aeruginosa from blood cultures, as well as respiratory specimens, constitutes strong evidence of true Pseudomonas pneumonia.

To help identify the best strategy for antibiotic therapy in Pseudomonas pneumonia, we evaluated the impact of empirical combination therapy on mortality in 100 consecutive patients with bacteremic $P$. aeruginosa pneumonia using a risk stratification model to adjust for potential differences.

\section{Methods}

\section{Data collection}

All patients $\geq 18$ years with $P$. aeruginosa bacteremic pneumonia between January 1997 and February 2011 were included. Patients with blood cultures positive for $P$. aeruginosa were identified from the computerized database of the clinical microbiology unit. Infectious diseases doctors then reviewed the medical records of these patients and collected demographic, clinical, and microbiological data. Ultimately, only patients with Pseudomonas pneumonia (see below) were included in the analysis. Patients with polymicrobial infections were excluded. The study was approved by the Asan Medical Center Institutional Review Board (S2012-1034-0001), and the requirement for patient consent was waved due to the retrospective nature of the study.

\section{Definitions}

Bacteremia was defined as $\geq 1$ positive blood culture for $P$. aeruginosa and the presence of the clinical features compatible with infection. If a patient had undergone recurrent episodes of $P$. aeruginosa bacteremia during the study period, only the first episode was considered. Pneumonia was defined as presence of: (1) new radiographic infiltration; (2) one or more of the following symptoms consistent with pneumonia (fever, cough, pleuritic chest pain, and dyspnea); and (3) isolation of $P$. aeruginosa from cultures of bronchoalveolar lavage (BAL) fluid or appropriate respiratory specimens [12,13]. The categories of pneumonia have been previously defined $[14,15]$. Empirical antibiotic therapy was considered adequate if therapy given intravenously within $48 \mathrm{~h}$ of the onset of pneumonia included antimicrobials to which the isolate was susceptible. Patients who received adequate treatment were stratified into 2 groups: a monotherapy group that received only 1 active antimicrobial, and a combination therapy group that simultaneously received 2 active antimicrobials [16]. Antimicrobial susceptibility testing was performed using the MicroScan system with the Neg Breakpoint Combo Panel 44 (Siemens Healthcare Diagnostics Inc., West
Sacramento, CA) according to standard criteria of the Clinical and Laboratory Standards Institute [17]. MDR was defined as resistance to more than three classes of antibiotics such as anti-pseudomonal beta-lactams, carbapenems, fluoroquinolones, and aminoglycosides [18].

\section{Statistical analysis}

All statistical analyses were performed using SAS software, version 9.1 (SAS Institute Inc, Cary, NC). Marginal structural models were used to estimate the difference in mortality between the adequate therapy group and the inadequate therapy group. Careful adjustment was made using weighted Cox proportional-hazards regression models that were based on the inverse-probability-oftreatment weighted (IPTW) method in order to reduce the effect of potential confounding factors and selection bias in this observational study [19]. IPTW estimation relies on multivariable logistic regression analysis. All variables with a $\mathrm{p}$ value $<0.2$ on univariable analysis were then introduced into the multivariable logistic regression model, and a backwards stepwise logistic regression was carried out. Sex, age, underlying diseases, multidrug resistance, McCabe score, APACHE II score, Pitt bacteremia score, clinical pulmonary infection score (CPIS), type of pneumonia, and use of previous antibiotics were classified as independent variables for the purposes of this study. $\mathrm{P}$ value $\leq 0.05$ was considered statistically significant.

\section{Results}

During the study period 1,361 patients with $P$. aeruginosa bacteremia were identified from our hospital records. One hundred (7.3\%) patients with concomitant pneumonia were eventually included in the analysis. The demographics, clinical features, microbiologic characteristics and clinical outcomes of these patients are shown in Table 1. Hematologic malignancy (34\%) was the most common underlying disease, followed by solid cancer (23\%). Of the 100 cases, 37 (37\%) originated in the community (18 community-acquired and 19 healthcareassociated infections). Thirty-eight (38.0\%) patients presented with septic shock. Sixty-five patients (65.0\%) were assigned to the adequate empirical therapy group, 32 (49.2\%) of whom received monotherapy and 33 (50.8\%) combination therapy. The antimicrobials used in monotherapy were as follows: ceftazidime $(n=10)$, cefepime $(\mathrm{n}=2)$, carbapenem $(\mathrm{n}=8)$, piperacillin-tazobactam $(\mathrm{n}=$ $7)$, and ciprofloxacin $(n=5)$. The remainder of the patients received combination therapy: piperacillintazobactam plus ciprofloxacin $(n=11)$, cefoperazonesulbactam plus aminoglycoside $(n=9)$, ceftazidime plus aminoglycoside $(\mathrm{n}=8)$, ceftazidime plus ciprofloxacin $(\mathrm{n}=3)$, and carbapenem plus ciprofloxacin $(\mathrm{n}=2)$. MDR pathogens were commonly found in the inadequate 
Table 1 Baseline characteristics of patients who received adequate antibiotic monotherapy and combination therapy, and inadequate therapy

\begin{tabular}{|c|c|c|c|c|c|}
\hline & \multirow[t]{2}{*}{$\begin{array}{c}\text { Total } \\
(\mathrm{n}=100)\end{array}$} & \multicolumn{2}{|c|}{$\begin{array}{l}\text { Adequate therapy } \\
\qquad(n=65)\end{array}$} & \multirow[t]{2}{*}{$\begin{array}{l}\text { Inadequate therapy } \\
\qquad(\mathrm{n}=35)\end{array}$} & \multirow[t]{2}{*}{$P$ value } \\
\hline & & $\begin{array}{l}\text { Monotherapy } \\
(\mathrm{n}=32)\end{array}$ & $\begin{array}{l}\text { Combination therapy } \\
(n=33)\end{array}$ & & \\
\hline Age (median years, range) & $59(53-67)$ & 60 & 56 & 61 & 0.24 \\
\hline Male gender & $74(74.0)$ & $27(84.4)$ & $24(72.7)$ & $23(65.7)$ & 0.22 \\
\hline \multicolumn{6}{|l|}{ Underlying disease } \\
\hline Hematologic malignancy & $34(34.0)$ & $12(37.5)$ & $14(42.4)$ & $8(22.9)$ & 0.21 \\
\hline Solid organ malignancy & $23(23.0)$ & $8(25.0)$ & $12(36.4)$ & $3(8.6)$ & 0.02 \\
\hline Neurologic disease & $14(14.0)$ & $7(21.9)$ & 0 & $7(20.0)$ & 0.01 \\
\hline Immunosuppression & $22(22.0)$ & $6(18.8)$ & $10(30.3)$ & $6(17.1)$ & 0.13 \\
\hline Structural lung disease & $8(8.0)$ & $2(6.3)$ & $4(12.1)$ & $2(5.9)$ & 0.65 \\
\hline Congestive heart failure & $9(9.0)$ & $2(6.3)$ & $1(3.0)$ & $6(17.1)$ & 0.13 \\
\hline Hemodialysis & $10(10.0)$ & $2(6.3)$ & $1(3.0)$ & $7(20.0)$ & 0.07 \\
\hline Liver cirrhosis & $5(5.0)$ & $1(3.1)$ & $2(6.1)$ & $2(5.7)$ & 0.99 \\
\hline Biliary disease & $2(2.0)$ & 0 & 0 & $2(5.7)$ & 0.33 \\
\hline McCabe score & & & & & $<0.001$ \\
\hline Non-fatal & $42(42.0)$ & $13(40.6)$ & $7(21.2)$ & $22(62.9)$ & \\
\hline Ultimately fatal & $44(44.0)$ & $18(56.3)$ & $20(60.6)$ & $6(17.1)$ & \\
\hline Rapidly fatal & $14(14.0)$ & $1(3.1)$ & $6(18.2)$ & $7(20.0)$ & \\
\hline APACHE II score, median (IQR) & $21(16-26)$ & $18.5(13-22)$ & $20(17-24.5)$ & $23(14-27)$ & 0.08 \\
\hline Pitt bacteremia score, median (IQR) & $3(1-5)$ & $2(0-4)$ & $3(2-5)$ & $4(2-6)$ & 0.05 \\
\hline CPIS, median (IQR) & $6(5-7)$ & $6(5-7)$ & $6(5-7)$ & $6(5-7)$ & 0.57 \\
\hline Type of pneumonia & & & & & 0.01 \\
\hline Community-acquired & $18(18.0)$ & $3(9.4)$ & $11(33.3)$ & $4(11.4)$ & \\
\hline Healthcare-associated & $19(19.0)$ & $8(25.0)$ & $6(18.2)$ & $5(14.3)$ & \\
\hline Hospital- acquired & $44(44.0)$ & $15(46.9)$ & $15(45.5)$ & $14(40.0)$ & \\
\hline Ventilator-associated & $19(19.0)$ & $6(18.8)$ & $1(3.0)$ & $12(34.3)$ & \\
\hline Initial manifestation within $24 \mathrm{~h}$ & & & & & 0.22 \\
\hline Bacteremia without SIRS & $6(6.0)$ & $2(6.3)$ & $2(6.1)$ & $2(5.7)$ & \\
\hline Sepsis & $35(35.0)$ & $17(53.1)$ & $8(24.2)$ & $10(28.6)$ & \\
\hline Severe sepsis & $21(21.0)$ & $6(18.8)$ & $7(21.2)$ & $8(22.9)$ & \\
\hline Septic shock & $38(38.0)$ & $7(21.9)$ & $16(48.5)$ & $15(42.9)$ & \\
\hline MDR-P. aeruginosa & $23(23.0)$ & $6(18.8)$ & $2(6.1)$ & $15(42.9)$ & 0.001 \\
\hline Previous antibiotic therapy & $55(55.0)$ & $17(30.9)$ & $11(20.0)$ & $27(49.1)$ & 0.009 \\
\hline Initial antimicrobial administration within $24 \mathrm{~h}$ & $94(94.0)$ & $29(90.6)$ & $32(96.9)$ & $33(94.3)$ & 0.60 \\
\hline Total duration of therapy, median days (IQR) & $14(8-21)$ & $15(9-19)$ & $14(8-22)$ & $14(7-21)$ & 0.71 \\
\hline Total length of hospital stay, median days (IQR) & $37.5(19-75)$ & 39 (19-94) & $28(13-60)$ & $52(26-82)$ & 0.10 \\
\hline $\begin{array}{l}\text { Length of hospital stay before bacteremia, } \\
\text { median days (IQR) }\end{array}$ & $12.5(0-39)$ & $9(0-29.5)$ & $0(0-19.5)$ & $29(9-59)$ & 0.005 \\
\hline $\begin{array}{l}\text { Length of hospital stay after bacteremia, } \\
\text { median days (IQR) }\end{array}$ & $16.5(7-36.5)$ & $18.5(10.8-52.5)$ & $16(6.5-34)$ & $17(7-33)$ & 0.21 \\
\hline \multicolumn{6}{|l|}{ Overall mortality } \\
\hline 7-day mortality & $21(21.0)$ & $5(15.6)$ & $6(18.2)$ & $10(28.6)$ & 0.382 \\
\hline 14-day mortality & $30(30.0)$ & $8(25.0)$ & $6(18.2)$ & $16(45.7)$ & 0.035 \\
\hline 28-day mortality & $51(51.0)$ & $17(53.1)$ & $10(30.3)$ & $24(68.6)$ & 0.01 \\
\hline
\end{tabular}

Data are presented as number (\%) unless otherwise specified.

$I Q R=$ interquartile range, $C P I S=$ Clinical Pulmonary Infection Score, $M D R=$ multidrug-resistant. 
therapy group (42.9\% [15 of 35] vs. 12.3\% [8 of 65], $p<$ $0.001)$. Among the 65 patients in the adequate therapy group, there was no significant difference in terms of the emergence of antimicrobial resistance between the monotherapy and combination therapy group (21.9\% [7/32] and $12.1 \%$ [4/33], respectively) $(p=0.29)$. However, the 2-week bacteria eradication rate $(54.5 \%$ [18/33] vs. $18.8 \%$ [6/32], $p=0.04$ ) and the 4-week eradication rate (54.5\% [18/33] vs. $28.1 \%$ [9/32], $p=0.04)$ were significantly higher in the combination therapy group than the monotherapy group. There were no subsequent episodes of Pseudomonas pneumonia after 1 month, but there were 6 subsequent episodes within the first 6 months (2 patients in the inadequate therapy group [5.7\%], 1 patient in adequate monotherapy group [3.1\%], and 3 patients in adequate combination therapy group [9.1\%]). However, there were no deaths due to recurrent $P$. aeruginosa infection, and all patients with subsequent episodes demonstrated full recoveries.

We identified 14 patients who had coinfection at the time of Pseudomonas pneumonia (6 patients in the inadequate therapy group and 8 patients in the adequate therapy group), though the rates of coinfection were not significantly different between the monotherapy group (9.4\% [3 of 32]) and the combination therapy group (15.2\% [5 of 33], $p=0.71$ ). Of these 14 patients, 8 patients died (4 patients in inadequate therapy, 2 patients in monotherapy, and 2 patients in the combination therapy group), but there were no deaths due to coinfection in either group.

The overall all-cause 28-day mortality was 51.0\% (51 of 100). The 28-day mortality was significantly higher in the inadequate therapy group than the adequate therapy group (68.6\% [24/35] vs. 41.5\% [27/65], $p=0.01)$. Variables such as sex, age, MDR, McCabe score, APACHE II score, Pitt bacteremia score, CPIS, and the use of previous antibiotics (which were determined to be significant by univariate analysis) were subjected to logistic regression modeling in order to identify the independent risk factors for all-cause mortality. Multivariate analysis indicated that the APACHE II score (adjusted odds ratio [AOR]: 1.08; 95\% confidence interval [CI]: 1.02-1.15; $p=0.01$ ) and inadequate therapy (AOR: 2.73 ; 95\% CI: $1.11-6.71 ; p=0.03)$ were independently associated with all-cause 28-day mortality (Additional file 1: Table S1). The marginal structural model was estimated using multiple logistic regression analysis. To reduce the effects of potential confounding factors and selection bias, additional analyses were performed with covariable adjustment using IPTW. These additional analyses showed that only inadequate therapy was significantly associated with 28-day mortality (AOR: 3.02; 95\% CI: $1.15-7.93 ; p=0.02)$ and that MDR was not a significant risk factor (data not shown).
To further examine the direct impact of combination therapy, we performed a subgroup analysis of the 65 patients who received adequate therapy. In univariate analysis, variables that were significantly associated with 28-day mortality included underlying hematologic malignancy, Pitt bacteremia score, initial clinical manifestation of bacteremia, and monotherapy. Multivariate logistic regression analysis identified the absence of septic shock at the time of bacteremia (AOR, 0.07; 95\% CI, 0.01-0.49; $p=0.008$ ), and combination therapy (AOR, 0.05; 95\% CI, $0.01-0.34 ; p=0.002$ ) as variables that were independently associated with decreased all-cause 28-day mortality (Table 2).

\section{Discussion}

In our present study, we evaluated the impact of empirical combination therapy on clinical outcomes in 100 consecutive patients with monomicrobial bacteremic Pseudomonas pneumonia. Adequate empirical combination therapy was associated with significantly lower 28day mortality.

Despite the high mortality rates in patients with $P$. aeruginosa pneumonia, it is still not clear how best to treat Pseudomonas pneumonia. Paul et al., in their metaanalysis of 64 randomized trials, compared the clinical outcomes of beta lactam monotherapy and beta lactamaminoglycoside combination therapy for the treatment of sepsis in immunocompetent patients [7]. In their study, beta lactam-aminoglycoside combination therapy was not associated with decreased mortality. On the other hand, Safdar and colleagues previously examined the impact of combination therapy on patients with gram-negative bacteremia and found a survival benefit associated with combination therapy in the subgroup of patients with $P$. aeruginosa bacteremia, demonstrating an approximately $50 \%$ reduction in mortality (OR: 0.50; 95\% CI: 0.32-0.79) [8]. These differing results may have been due to the limitations of the earlier studies, including: (1) the combinations of different pathogens reported; (2) the greatly varying proportion of patients with pneumonia (range: 14\%-100\%); (3) the use of aminoglycoside as a monotherapy, which is not currently considered an appropriate therapy for $P$. aeruginosa infection; and (4) the possibility of $P$. aeruginosa colonization of the respiratory tract was not excluded. To overcome these limitations, we included a large number of consecutive patients with monomicrobial bacteremic Pseudomonas pneumonia in our study.

In our present analyses, adequate empirical therapy was associated with a significant reduction in mortality after 7 days. Analysis of the adequate empirical therapy group revealed that combination therapy was a variable independently associated with decreased 28-day mortality. One possible explanation for the decreased mortality 
Table 2 Logistic regression analysis of the risk factors for 28-day mortality in patients with adequate empirical therapy

\begin{tabular}{|c|c|c|c|c|}
\hline \multirow[t]{2}{*}{ Variable } & \multirow{2}{*}{$\begin{array}{l}\begin{array}{l}\text { Univariate } \\
\text { analysis }\end{array} \\
\text { OR }(95 \% \mathrm{Cl})\end{array}$} & \multirow[t]{2}{*}{$P$} & \multirow{2}{*}{$\begin{array}{l}\text { Multivariate } \\
\text { analysis } \\
\text { OR }(95 \% \mathrm{CI})\end{array}$} & \multirow[t]{2}{*}{$P$} \\
\hline & & & & \\
\hline Age & $1.00(0.96-1.04)$ & .98 & & \\
\hline Male gender & $0.48(0.14-1.76)$ & .27 & & \\
\hline \multicolumn{5}{|l|}{ Underlying disease } \\
\hline Solid organ malignancy & $1.22(0.42-3.56)$ & .71 & & \\
\hline $\begin{array}{l}\text { Hematologic } \\
\text { malignancy }\end{array}$ & $2.33(0.84-6.46)$ & .10 & & \\
\hline Structural lung disease & $0.25(0.03-2.31)$ & .22 & & \\
\hline Neurologic disease & $0.21(0.02-1.81)$ & .15 & & \\
\hline Congestive heart failure & $2.96(0.26-34.42)$ & .39 & & \\
\hline Hemodialysis & $0.69(0.06-8.05)$ & .77 & & \\
\hline Immunosuppression & $0.62(0.20-1.93)$ & .41 & & \\
\hline \multicolumn{5}{|l|}{ McCabe score } \\
\hline Non-fatal & $0.57(0.09-3.38)$ & .54 & & \\
\hline Ultimately fatal & $1.20(0.24-6.11)$ & .83 & & \\
\hline Rapidly fatal & 1.0 (referent) & & & \\
\hline APACHE II score & $1.04(0.97-1.11)$ & .32 & & \\
\hline Pitt bacteremia score & $1.21(0.96-1.51)$ & .10 & & \\
\hline CPIS & $1.06(0.73-1.54)$ & .77 & & \\
\hline \multicolumn{5}{|l|}{ Type of pneumonia } \\
\hline Community-acquired & $1.00(0.16-6.26)$ & .99 & & \\
\hline Healthcare-associated & $0.74(1.12-4.73)$ & .75 & & \\
\hline Hospital- acquired & $1.02(0.19-5.37)$ & .98 & & \\
\hline Ventilator-associated & 1.0 (referent) & & & \\
\hline MDR-P. aeruginosa & $0.43(0.08-2.30)$ & .32 & & \\
\hline Previous antibiotic therapy & $1.42(0.53-3.86)$ & .49 & & \\
\hline \multicolumn{5}{|c|}{ Initial manifestation within $24 \mathrm{~h}$} \\
\hline Sepsis & $0.36(0.11-1.17)$ & .09 & $0.07(0.01-0.49)$ & 0.008 \\
\hline Severe sepsis & $0.29(0.07-1.21)$ & .09 & $0.13(0.02-0.89)$ & 0.04 \\
\hline Septic shock & 1.0 (referent) & & & \\
\hline \multicolumn{5}{|c|}{ Type of adequate empirical therapy } \\
\hline Monotherapy & $0.38(0.14-1.06)$ & .06 & $0.05(0.01-0.34)$ & 0.002 \\
\hline Combination therapy & 1.0 (referent) & & & \\
\hline
\end{tabular}

$M D R=$ multidrug-resistant.

could be a reduced frequency of emergence of antimicrobial resistance. However, there was no significant difference between the emergence of antimicrobial resistance in the monotherapy group $(21.9 \%[7 / 32])$ and the combination therapy group $(12.1 \%[4 / 33], p=0.29)$. Even in cases in where antimicrobial resistance emerged, only 3 patients ( 3 of 7) and 1 patient ( 1 of 4 ) died, respectively. Thus, the emergence of resistance may not affect the mortality rate. In contrast, the 2-week (18.8\% vs. $54.5 \%, p=0.04)$ and 4-week bacteria eradication rates (28.1\% vs. $54.5 \%, p=0.04)$ after bacteremia were significantly higher in the combination therapy group than in the monotherapy group. Because there was no significant difference in terms of the emergence of antimicrobial resistance between the monotherapy and combination therapy groups, these findings suggest that empirical combination therapy may contribute to the reduction in mortality by accelerating eradication. Hence, the manner in which combination therapy decreases mortality in patients with $P$. aeruginosa infection is an interesting question. Unfortunately, we did not perform a prospective study that could evaluate this and future analyses of this type will be required to do so.

The present study has some limitations. First, it was retrospective and performed at a single center, so there is the potential for bias that would limit the ability to draw a firm conclusion about the direct impact of antimicrobial therapy. However, it has been shown that elaborate statistical methods, such as the marginal structural model using IPTW, provide a better method of control for confounding influences by improving the adjustment of differences between treatment groups [20]. Furthermore, we tried to evaluate the possible factors that could affect the clinical outcomes of patients with pneumonia as much as possible. Second, our study included only patients with bacteremia, so our finding may not be directly applicable to Pseudomonas pneumonia without bacteremia.

\section{Conclusions}

In conclusion, our data strongly suggest that adequate empirical combination therapy can decrease mortality in patients with bacteremic Pseudomonas pneumonia. Additional prospective studies are therefore warranted.

\section{Additional file}

Additional file 1: Table S1. Logistic regression analysis of the risk

factors for 28-day mortality in all patients.

\section{Competing interests}

The authors declare that they have no competing interests.

\section{Authors' contributions}

Conceived and designed the study: SYP, SOL, SHC; Performed the study: SYP, HJP, SMM. Analyzed the data: SYP, KHP. YPC. Contributed reagents/materials/ analysis tools: SYP, MNK, SHK, YSK, JHW. Wrote the paper: SYP, SHC. Critical review of the paper: All. All authors read and approved the final manuscript.

\section{Author details}

${ }^{1}$ Departments of Infectious Diseases, Asan Medical Center, University of Ulsan College of Medicine, 388-1 Pungnap-dong, Songpa-gu, Seoul, Republic of Korea. ${ }^{2}$ Departments of Laboratory Medicine, Asan Medical Center, University of Ulsan College of Medicine, 388-1 Pungnap-dong, Songpa-gu, Seoul,

Republic of Korea. 
Received: 2 March 2012 Accepted: 15 November 2012

Published: 16 November 2012

\section{References}

1. Joo EJ, Kang Cl, Ha YE, Park SY, Kang SJ, Wi YM, Lee NY, Chung DR, Peck KR, Song $\mathrm{JH}$ : Impact of inappropriate empiric antimicrobial therapy on outcome in Pseudomonas aeruginosa bacteraemia: a stratified analysis according to sites of infection. Infection 2011, 39(4):309-318.

2. Fujitani S, Sun HY, Yu VL, Weingarten JA: Pneumonia due to Pseudomonas aeruginosa: part I: epidemiology, clinical diagnosis, and source. Chest 2011, 139(4):909-919.

3. National Nosocomial Infections Surveillance System: National Nosocomial Infections Surveillance (NNIS) System Report, data summary from January 1992 through June 2004, issued October 2004. Am J Infect Control 2004, 32(8):470-485.

4. Sun HY, Fujitani S, Quintiliani R, Yu VL: Pneumonia due to Pseudomonas aeruginosa: part II: antimicrobial resistance, pharmacodynamic concepts, and antibiotic therapy. Chest 2011, 139(5):1172-1185.

5. El Solh AA, Alhajhusain A: Update on the treatment of Pseudomonas aeruginosa pneumonia. J Antimicrob Chemother 2009, 64(2):229-238.

6. Anderson ET, Young LS, Hewitt WL: Antimicrobial synergism in the therapy of gram-negative rod bacteremia. Chemotherapy 1978, 24(1):45-54

7. Paul M, Benuri-Silbiger I, Soares-Weiser K, Leibovici L: Beta lactam monotherapy versus beta lactam-aminoglycoside combination therapy for sepsis in immunocompetent patients: systematic review and metaanalysis of randomized trials. BMJ 2004, 328(7441):668-681.

8. Safdar N, Handelsman J, Maki DG: Does combination antimicrobial therapy reduce mortality in Gram-negative bacteraemia? A metaanalysis. Lancet Infect Dis 2004, 4(8):519-527.

9. Traugott KA, Echevarria K, Maxwell P, Green K, Lewis JS 2nd: Monotherapy or combination therapy? The Pseudomonas aeruginosa conundrum. Pharmacotherapy 2011, 31(6):598-608.

10. Heyland DK, Dodek P, Muscedere J, Day A, Cook D, Canadian Critical Care Trials Group: Randomized trial of combination versus monotherapy for the empiric treatment of suspected ventilator-associated pneumonia. Crit Care Med 2008, 36(3):737-744.

11. Chamot E, Boffi El Amari E, Rohner P, Van Delden C: Effectiveness of combination antimicrobial therapy for Pseudomonas aeruginosa bacteremia. Antimicrob Agents Chemother 2003, 47(9):2756-2764.

12. Carratalà J, Mykietiuk A, Fernández-Sabé N, Suárez C, Dorca J, Verdaguer R, Manresa F, Gudiol F: Health care-associated pneumonia requiring hospital admission: epidemiology, antibiotic therapy, and clinical outcomes. Arch Intern Med 2007, 167(13):1393-1399.

13. Mandell LA, Wunderink RG, Anzueto A, Bartlett JG, Campbell GD, Dean NC, Dowell SF, File TM Jr, Musher DM, Niederman MS, Torres A, Whitney CG, Infectious Diseases Society of America; American Thoracic Society: Infectious Diseases Society of America/American Thoracic Society consensus guidelines on the management of community-acquired pneumonia in adults. Clin Infect Dis 2007, 44:S27-S72.

14. Kollef MH, Shorr A, Tabak YP, Gupta V, Liu LZ, Johannes RS: Epidemiology and outcomes of health-care-associated pneumonia: results from a large US database of culture-positive pneumonia. Chest 2005, 128(6):3854-3862.

15. American Thoracic Society; Infectious Diseases Society of America: Guidelines for the management of adults with hospital-acquired, ventilator-associated, and healthcare-associated pneumonia. Am J Respir Crit Care Med 2005, 171(4):388-416.

16. Garnacho-Montero J, Sa-Borges M, Sole-Violan J, Barcenilla F, EscorescaOrtega A, Ochoa M, Cayuela A, Rello J: Optimal management therapy for Pseudomonas aeruginosa ventilator-associated pneumonia: an observational, multicenter study comparing monotherapy with combination antibiotic therapy. Crit Care Med 2007, 35(8):1888-1895.

17. Clinical and Laboratory Standards Institute: Performance standards for antimicrobial testing: seventeenth informational supplement. CLSI document M100-S17. Wayne, PA: Clinical and Laboratory Standards Institute; 2007.
18. Magiorakos AP, Srinivasan A, Carey RB, Carmeli Y, Falagas ME, Giske CG, Harbarth S, Hindler JF, Kahlmeter G, Olsson-Liljequist B, Paterson DL, Rice LB, Stelling J, Struelens MJ, Vatopoulos A, Weber JT, Monnet DL: Multidrugresistant, extensively drug-resistant and pandrug-resistant bacteria: an international expert proposal for interim standard definitions for acquired resistance. Clin Microbiol Infect 2012, 18(3):268-281.

19. Robins JM, Hernán MA, Brumback B: Marginal structural models and causal inference in epidemiology. Epidemiology 2000, 11(5):550-560.

20. Suarez D, Haro JM, Novick D, Ochoa S: Marginal structural models might overcome confounding when analyzing multiple treatment effects in observational studies. J Clin Epidemiol 2008, 61(6):525-530.

doi:10.1186/1471-2334-12-308

Cite this article as: Park et al:: Impact of adequate empirical combination therapy on mortality from bacteremic Pseudomonas aeruginosa pneumonia. BMC Infectious Diseases 2012 12:308.

\section{Submit your next manuscript to BioMed Central and take full advantage of:}

- Convenient online submission

- Thorough peer review

- No space constraints or color figure charges

- Immediate publication on acceptance

- Inclusion in PubMed, CAS, Scopus and Google Scholar

- Research which is freely available for redistribution

Submit your manuscript at www.biomedcentral.com/submit
C) BioMed Central 